Supporting Information for:

\title{
Effects of Strong Electronic Coupling in Chlorin and Bacteriochlorin Dyads
}

Hyun Suk Kang, ${ }^{\dagger}$ Nopondo N. Esemoto, ${ }^{\ddagger}$ James R. Diers, ${ }^{\S}$ Dariusz M. Niedzwiedzki, ${ }^{\#}$ Jordan A. Greco," Joshua Akhigbe, ${ }^{\star}$ Zhanqian Yu, ${ }^{\ddagger}$ Chirag Pancholi, ${ }^{\ddagger}$ Ganga Viswanathan Bhagavathy, ${ }^{\ddagger}$ Jamie K. Nguyen, ${ }^{\ddagger}$ Christine Kirmaier, ${ }^{\dagger}$ Robert R. Birge, ${ }^{*, l}$ Marcin Ptaszek, ${ }^{*}$, Dewey Holten, ${ }^{* \dagger}$ and David F. Bocian ${ }^{*}, \S$

${ }^{\dagger}$ Department of Chemistry, Washington University, St. Louis, Missouri 63130-4889, United States

${ }^{*}$ Department of Chemistry and Biochemistry, University of Maryland, Baltimore County, Baltimore, Maryland 21250-0001, United States

${ }^{\S}$ Department of Chemistry. University of California, Riverside, California 92521-0403, United States

"Photosynthetic Antenna Research Center, Washington University, St. Louis, Missouri 631304889, United States

'Department of Chemistry, University of Connecticut, Storrs, Connecticut 06269-3060, United States

\section{Table of Contents}

Topic

Page

1. Syntheses of Dyads and Benchmarks S1-S4

2. Absorption and Fluorescence Spectra of Dyads and Benchmarks in Benzonitrile S5-S6

3. Resolution Enhanced Absorption Spectra of Dyads S7

4. $\mathrm{MO}$ characteristics of $\mathbf{B C}_{2}-\boldsymbol{\beta}_{3} \mathbf{B}$ S8

5. Full Citations for References with Over Ten Authors S9

\section{Syntheses of Dyads and Benchmarks}

General Experimental Section. ${ }^{1} \mathrm{H}$ NMR spectra $(400 \mathrm{MHz})$ and ${ }^{13} \mathrm{C}$ NMR $(100 \mathrm{MHz})$ spectra were collected at room temperature in $\mathrm{CDCl}_{3}$ unless noted otherwise. Chemical shifts $(\delta)$ were callibrated using solvent peaks $\left({ }^{1} \mathrm{H}\right.$ signals: residual proton signals: $7.26 \mathrm{ppm}$ for chloroform, ${ }^{13} \mathrm{C}$ signals: 77.0 for $\mathrm{CDCl}_{3}$ ). All solvents and commercialy available reagents were used as received. Palladium coupling reactions was performed using commercially available anhydrous solvents (toluene and DMF). Known compounds $1,{ }^{45} 3,{ }^{47}$ and $4^{47}$ were synthesized following reported procedures.

General procedure for Sonogashira cross-coupling reactions. Schlenk flask was charged with all solid reagents and (o-tol) $)_{3} \mathrm{P}$ (if required), and solvent was added. The resulting mixture was degassed by freeze-thaw cycles (two times), and filled with nitrogen. A palladium catalyst was added to the reaction flask, and the mixture was degassed again by freeze-thaw 
cycles (two times), and filled with nitrogen. If TIPS-ethynyl, TMS-ethynyl, or phenyl acetylene were used as reagents, they were added after final degassing cycle. Reaction flask was warmed to the room temperature and placed in the pre-heated oil bath $\left(60{ }^{\circ} \mathrm{C}\right)$. The progress of the reaction was monitored by TLC, UV-vis spectroscopy, and LD-MS. When consumption of the hydroporphyrin starting material was determined, the reaction mixture was diluted with dichloromethane, washed (water and brine), dried $\left(\mathrm{Na}_{2} \mathrm{SO}_{4}\right)$, and concentrated. The final purification was done by column chromatography, as specified below.

15-Ethynyl-18,18-dimethyl-10-(4-methylphenyl)chlorin (2). Samples of 1 (30.0 mg. $0.0600 \mathrm{mmol})$, TMS-ethynyl $(30 \mu \mathrm{L}, 0.090 \mathrm{mmol}), \mathrm{Pd}_{2}(\mathrm{dba})_{3}(11.0 \mathrm{mg}, 0.0120 \mathrm{mmol}),(o \text {-tol })_{3} \mathrm{P}$ (21.4 mg, $0.0703 \mathrm{mmol})$, in toluene/ $\mathrm{Et}_{3} \mathrm{~N}(25 \mathrm{~mL}, 5: 1)$, were reacted overnight as described in General Procedure. Column chromatography (silica, hexanes $/ \mathrm{CH}_{2} \mathrm{Cl}_{2}, 2: 1$ ) afforded a mixture of 18,18-dimethyl-10-(4-methylphenyl)-15-[(2-trimethylsilyl)ethynyl]chlorin with an unknown byproduct. ${ }^{1} \mathrm{H}$ NMR $\left(\mathrm{CDCl}_{3}, 400 \mathrm{MHz}\right) \delta-1.64(\mathrm{bs}, 1 \mathrm{H}),-1.45(\mathrm{bs}, 1 \mathrm{H}), 0.55(\mathrm{~s}, 9 \mathrm{H}), 2.05(\mathrm{~s}, 6 \mathrm{H})$, 2.69 (s, 3H), 4.69 (s, 2H), $7.53(\mathrm{~d}, J=7.7 \mathrm{~Hz}, 2 \mathrm{H}), 8.00$ (d, $J=7.7 \mathrm{~Hz}, 2 \mathrm{H}), 8.56(\mathrm{~d}, J=4.3 \mathrm{~Hz}$, $1 \mathrm{H}), 8.75(\mathrm{~d}, J=4.8 \mathrm{~Hz}, 1 \mathrm{H}), 8.80(\mathrm{~s}, 1 \mathrm{H}), 8.86(\mathrm{~d}, J=4.3 \mathrm{~Hz}, 2 \mathrm{H}$, two overlapped doublets), $9.14(\mathrm{~d}, J=4.7 \mathrm{~Hz}, 1 \mathrm{H}), 9.20(\mathrm{~d}, J=4.8 \mathrm{~Hz}, 1 \mathrm{H}), 9.71(\mathrm{~s}, 1 \mathrm{H}) ; \mathrm{MS} m / z[\mathrm{M}+\mathrm{H}]^{+}$Calcd for $\mathrm{C}_{34} \mathrm{H}_{34} \mathrm{~N}_{4} \mathrm{Si}$ 527.2626; Found (HRMS-ESI) 527.2623. The resulting mixture was dissolved in THF/MeOH $(8 \mathrm{~mL}, 1: 1)$, treated with $\mathrm{K}_{2} \mathrm{CO}_{3}(4.9 \mathrm{mg}, 0.036 \mathrm{mmol})$, and stirred at the room temperature for $4 \mathrm{~h}$. The reaction mixture was diluted with $\mathrm{CH}_{2} \mathrm{Cl}_{2}$, washed (water, brine), dried $\left(\mathrm{Na}_{2} \mathrm{SO}_{4}\right)$, and concentrated. Column chromatography (silica, hexane/ethyl acetate, 9:1), afforded a pinkish powder $(9.1 \mathrm{mg}, 33 \%) .{ }^{1} \mathrm{H}$ NMR $\left(\mathrm{CDCl}_{3}, 400 \mathrm{MHz}\right) \delta-1.64$ (bs, $\left.1 \mathrm{H}\right),-1.45(\mathrm{bs}, 1 \mathrm{H})$, 0.55 (s, 9H), 2.05 (s, 6H), 2.69 (s, 3H), $3.98(\mathrm{~s}, 1 \mathrm{H}), 4.73(\mathrm{~s}, 2 \mathrm{H}), 7.53$ (d, $J=8.1 \mathrm{~Hz}, 2 \mathrm{H}), 8.00$ $(\mathrm{d}, J=8.1 \mathrm{~Hz}, 2 \mathrm{H}), 8.56(\mathrm{~d}, J=4.3 \mathrm{~Hz}, 1 \mathrm{H}), 8.76(\mathrm{~d}, J=4.8 \mathrm{~Hz}, 1 \mathrm{H}), 8.81(\mathrm{~s}, 1 \mathrm{H}), 8.87$ (d, $J=$ $4.3 \mathrm{~Hz}, 2 \mathrm{H}$, two overlapped doublets), 9.16 (d, $J=4.7 \mathrm{~Hz}, 1 \mathrm{H}), 9.22$ (d, $J=4.8 \mathrm{~Hz}, 1 \mathrm{H}), 9.73$ (s, $1 \mathrm{H})$. MS $m / z[\mathrm{M}+\mathrm{H}]^{+}$Calcd for $\mathrm{C}_{31} \mathrm{H}_{26} \mathrm{~N}_{4}$ 455.2230; Found (HRMS-ESI) 455.2234.

18,18-Dimethyl-10-(4-methylphenyl)-15-(triisopropylsilylethynyl)chlorin $\quad$ (C-mB). Sample of 1 (40.0 mg, $0.0785 \mathrm{mmol})$, TIPS-ethynyl $(53 \mu \mathrm{L}, 0.236 \mathrm{mmol}), \operatorname{Pd}_{2}(\mathrm{dba})_{3}(14.6 \mathrm{mg}$, $0.0160 \mathrm{mmol}),(o \text {-tol) })_{3} \mathrm{P}(28.7 \mathrm{mg}, 0.0943 \mathrm{mmol})$, in toluene/ $\mathrm{Et}_{3} \mathrm{~N}(30 \mathrm{~mL}, 5: 1)$, were reacted as described in General Procedure. Column chromatography (silica, hecanes $/ \mathrm{CH}_{2} \mathrm{Cl}_{2} 3: 1$ ) provides a mixture of products which was further purified using preparative TLC (silica, hexanes/ethyl acetate 100:1), the green spot with higher $\mathrm{R}_{\mathrm{f}}$ value was collected to afford a pinkish powder, 10.1 $\mathrm{mg}, 21 \% .{ }^{1} \mathrm{H}$ NMR $\left(\mathrm{CDCl}_{3}, 400 \mathrm{MHz}\right) \delta-1.66(\mathrm{bs}, 1 \mathrm{H}),-1.41(\mathrm{bs}, 1 \mathrm{H}), 1.41-1.47(\mathrm{~m}, 21 \mathrm{H})$, $2.08(\mathrm{~s}, 6 \mathrm{H}), 2.70(\mathrm{~s}, 3 \mathrm{H}), 4.76(\mathrm{~s}, 2 \mathrm{H}), 7.55$ (d, $J=7.6 \mathrm{~Hz}, 2 \mathrm{H}), 8.03(\mathrm{~d}, J=7.6 \mathrm{~Hz}, 2 \mathrm{H}), 8.60$ (d, $J=4.3 \mathrm{~Hz}, 1 \mathrm{H}), 8.82(\mathrm{~d}, J=4.7 \mathrm{~Hz}, 1 \mathrm{H}), 8.83(\mathrm{~s}, 1 \mathrm{H}), 8.86(\mathrm{~d}, J=4.6 \mathrm{~Hz}, 1 \mathrm{H}), 8.88(\mathrm{~d}, J=4.3$ $\mathrm{Hz}, 1 \mathrm{H}), 9.14(\mathrm{~d}, J=4.6 \mathrm{~Hz}, 1 \mathrm{H}), 9.32(\mathrm{~d}, J=4.7 \mathrm{~Hz}, 1 \mathrm{H}), 9.72(\mathrm{~s}, 1 \mathrm{H}) .{ }^{13} \mathrm{C} \mathrm{NMR}\left(\mathrm{CDCl}_{3}, 100\right.$ MHz) $\delta 11.9,19.2,21.7,31.7,45.9,53.2,93.4,95.0,96.6,108.2,109.2,123.0,123.7,123.8$, 127.7, 128.7, 129.0, 132.3, 132.8, 134.1, 134.9, 135.3, 137.5, 138.8, 141.3, 141.8, 151.7, 152.8, 167.2, 170.1. MS m/z [M+H] $]^{+}$Calcd for $\mathrm{C}_{40} \mathrm{H}_{46} \mathrm{~N}_{4} \mathrm{Si}$ 611.3564; Found (HRMS-ESI) 611.3569.

18,18-Dimethyl-10-(4-methylphenyl)-15-phenylethynylchlorin $(\mathrm{C}-\mathrm{mE})$. Samples of $\mathbf{1}$ (16 mg, $0.031 \mathrm{mmol})$, phenyl acetylene $(2.0 \mu \mathrm{L}, 0.62 \mathrm{mmol}),\left(\operatorname{Pd}_{2}(\mathrm{dba})_{3}(4.3 \mathrm{mg}, 0.0047 \mathrm{mmol})\right.$, 
$(o \text {-tol) })_{3} \mathrm{P}(11.3 \mathrm{mg}, 0.0371 \mathrm{mmol})$, in toluene $/ \mathrm{Et}_{3} \mathrm{~N}(20 \mathrm{~mL}, 5: 1)$, were reacted overnight as described in General procedure. Column chromatography (silica, hexanes/dichloromethane 2:1 and second column hexanes/ethyl acetate 15:1) afforded a maroon solid (9.2 mg, 56\%). ${ }^{1} \mathrm{H}$ NMR $\left(\mathrm{CDCl}_{3}, 400 \mathrm{MHz}\right) \delta-1.65(\mathrm{bs}, 1 \mathrm{H}),-1.39(\mathrm{bs}, 1 \mathrm{H}), 2.07(\mathrm{~s}, 6 \mathrm{H}), 2.69(\mathrm{~s}, 3 \mathrm{H}), 4.81(\mathrm{~s}, 2 \mathrm{H}), 7.43-$ $7.48(\mathrm{~m}, 1 \mathrm{H}), 7.51-7.55(\mathrm{~m}, 4 \mathrm{H}), 7.91-7.94(\mathrm{~m}, 2 \mathrm{H}), 8.00-8.03(\mathrm{~m}, 2 \mathrm{H}), 8.56(\mathrm{~d}, J=4.3 \mathrm{~Hz}, 1 \mathrm{H})$, $8.78(\mathrm{~d}, J=4.8 \mathrm{~Hz}, 1 \mathrm{H}), 8.82(\mathrm{~s}, 1 \mathrm{H}), 8.87(\mathrm{~d}, J=4.3 \mathrm{~Hz}$, two overlapped doublets, $2 \mathrm{H}), 9.14$ (d, $J=4.6 \mathrm{~Hz}, 1 \mathrm{H}), 9.30(\mathrm{~d}, J=4.8 \mathrm{~Hz}, 1 \mathrm{H}), 9.71(\mathrm{~s}, 1 \mathrm{H}) .{ }^{13} \mathrm{C} \mathrm{NMR}\left(\mathrm{CDCl}_{3}, 100 \mathrm{MHz}\right) \delta 21.7,31.6$, 46.0, 52.9, 92.3, 93.1, 95.2, 95.4, 108.2, 122.8, 123.7, 123.9, 124.5, 127.7, 128.2, 128.6, 128.8, 131.6, 132.4, 132.8, 134.1, 134.9, 135.3, 137.6, 138.8, 141.2, 141.3, 151.8, 152.9, 166.8, 176.0. MS $m / z[\mathrm{M}+\mathrm{H}]^{+}$Calcd for $\mathrm{C}_{37} \mathrm{H}_{30} \mathrm{~N}_{4} 531.2543$; Found (HRMS-ESI) 531.2541.

$\mathbf{C}_{2}-\boldsymbol{m}$ B. Samples of $2(5.0 \mathrm{mg}, 0.011 \mathrm{mmol}), \mathrm{Pd}_{2}(\mathrm{dba})_{3}(3.0 \mathrm{mg}, 0.0033 \mathrm{mmol}),(o-\mathrm{tol})_{3} \mathrm{P}$ $(4.0 \mathrm{mg}, 0.0132 \mathrm{mmol})$, in toluene/ $\mathrm{Et}_{3} \mathrm{~N}(6 \mathrm{~mL}, 5: 1)$, were reacted for $2 \mathrm{hrs}$ as described in General procedure. Column chromatography (silica, hexanes/dichloromethane $3: 1 \rightarrow 2: 1$ ) afforded a greenish solid (1.2 mg, 25\%). ${ }^{1} \mathrm{H}$ NMR $\left(\mathrm{CDCl}_{3}, 400 \mathrm{MHz}\right) \delta-1.39$ (bs, $\left.2 \mathrm{H}\right),-1.09$ (bs, 2H), $2.13(\mathrm{~s}, 12 \mathrm{H}), 2.70(\mathrm{~s}, 6 \mathrm{H}), 4.97(\mathrm{~s}, 4 \mathrm{H}), 7.56(\mathrm{~d}, J=8.0 \mathrm{~Hz}, 4 \mathrm{H}), 8.04(\mathrm{~d}, J=8.0 \mathrm{~Hz}, 4 \mathrm{H})$, $8.56(\mathrm{~d}, J=4.3 \mathrm{~Hz}, 2 \mathrm{H}), 8.84(\mathrm{~d}, J=4.3 \mathrm{~Hz}, 2 \mathrm{H}), 8.84(\mathrm{~s}, 2 \mathrm{H}), 8.86-8.87$ (two overlapped doublets, $4 \mathrm{H}), 9.16$ (d, $J=4.9 \mathrm{~Hz}, 2 \mathrm{H}), 9.44(\mathrm{~d}, J=4.9 \mathrm{~Hz}, 2 \mathrm{H}), 9.72(\mathrm{~s}, 2 \mathrm{H}) . \mathrm{MS} m / z[\mathrm{M}+\mathrm{H}]^{+}$ Calcd for $\mathrm{C}_{62} \mathrm{H}_{50} \mathrm{~N}_{8}$ 907.4231; Found (HRMS-ESI) 907.4233.

$\mathbf{C}_{2}$ - $\boldsymbol{m E}$ E. Samples of $1(5.6 \mathrm{mg}, 0.011 \mathrm{mmol}), 2(5.0 \mathrm{mg}, 0.011 \mathrm{mmol}), \mathrm{Pd}_{2}(\mathrm{dba})_{3}(2.0 \mathrm{mg}$, $0.0022 \mathrm{mmol}),(o \text {-tol })_{3} \mathrm{P}(4.0 \mathrm{mg}, 0.0132 \mathrm{mmol})$, in toluene/Et ${ }_{3} \mathrm{~N}(6 \mathrm{~mL}, 5: 1)$, were reacted overnight as described in General procedure. Column chromatography (silica, hexanes/dichloromethane $2: 1 \rightarrow 1: 1)$ afforded a green solid, which was washed with $\mathrm{MeOH}$ to provide a green powder $(3.3 \mathrm{mg}, 34 \%) .{ }^{1} \mathrm{H}$ NMR $\left(\mathrm{CDCl}_{3}, 400 \mathrm{MHz}\right) \delta-1.49$ (bs, $\left.2 \mathrm{H}\right),-1.17$ (bs, 2H), 2.20 (s, 12H), 2.71 (s, 6H), 5.27 (s, 4H), 7.58 (d, $J=7.7 \mathrm{~Hz}, 4 \mathrm{H}), 8.09$ (d, $J=7.7 \mathrm{~Hz}, 4 \mathrm{H})$, $8.62(\mathrm{~d}, J=4.3 \mathrm{~Hz}, 2 \mathrm{H}), 8.90(\mathrm{~d}, J=4.3 \mathrm{~Hz}, 2 \mathrm{H}), 8.92(\mathrm{~s}, 2 \mathrm{H}), 8.92$ (d, overlapped with singlet, 2H), 8.94 (d, $J=4.7 \mathrm{~Hz}, 2 \mathrm{H}), 9.19$ (d, $J=4.7 \mathrm{~Hz}, 2 \mathrm{H}), 9.76$ (s, 2H), 9.80 (d, $J=4.7 \mathrm{~Hz}, 2 \mathrm{H}) . \mathrm{MS}$ $\mathrm{m} / z[\mathrm{M}+\mathrm{H}]^{+}$Calcd for $\mathrm{C}_{60} \mathrm{H}_{50} \mathrm{~N}_{8}$ 883.4231; Found (HRMS-ESI) 883.4260.

5-Methoxy-8,8,18,18-tetramethyl-15-phenylethynylbacteriochlorin

$(\mathbf{B C}-\boldsymbol{m E} \mathbf{E})$. Samples of $3(6.9 \mathrm{mg}, 0.0146 \mathrm{mmol})$, phenyl acetylene $(2.0 \mu 1,0.018 \mathrm{mmol}), \mathrm{Pd}_{2}\left(\mathrm{dba}_{3}(2.6 \mathrm{mg}\right.$, $0.0028 \mathrm{mmol}),(o \text {-tol })_{3} \mathrm{P}(5.3 \mathrm{mg}, 0.0175 \mathrm{mmol})$, in toluene/Et ${ }_{3} \mathrm{~N}(6 \mathrm{~mL}, 5: 1)$, were reacted overnight as described in General procedure. Column chromatography (silica, hexanes/ethyl acetate 20:1) afforded a pink solid (2.1 mg, 29\%). ${ }^{1} \mathrm{H}$ NMR $\left(\mathrm{CDCl}_{3}, 400 \mathrm{~Hz}\right) \delta-1.87(\mathrm{bs}, 1 \mathrm{H}),-$ 1.59 (bs, 1H), 1.96 (s, 6H), 1.97 (s, 6H), 4.37 (s, 2H), 4.48 (s, 3H), 4.63 (s, 2H), 7.47-7.42 (m, $1 \mathrm{H}), 7.54-7.50(\mathrm{~m}, 2 \mathrm{H}), 7.92-7.98(\mathrm{~m}, 2 \mathrm{H}), 8.63(\mathrm{~s}, 1 \mathrm{H}), 8.63$ (dd, overlapped with singlet, $1 \mathrm{H})$, 8.66 (s, 1H), 8.74 (dd, $J=1.9 \mathrm{~Hz}, J=4.5 \mathrm{~Hz}, 1 \mathrm{H}), 8.89$ (dd, $J=2.0 \mathrm{~Hz}, J=4.5 \mathrm{~Hz}, 1 \mathrm{H}), 9.16$ (dd, $J=1.9 \mathrm{~Hz}, J=4.5 \mathrm{~Hz}, 1 \mathrm{H}$ ). MS $m / z[\mathrm{M}]^{+}$Calcd for $\mathrm{C}_{33} \mathrm{H}_{32} \mathrm{~N}_{4} \mathrm{O} 500.2571$; Found (HRMSESI) 500.2570 .

$\mathbf{B C}_{2}-\boldsymbol{m} \mathbf{E}$. Samples of $3(2.3 \mathrm{mg}, 0.0047 \mathrm{mmol}), \mathbf{B C}-\boldsymbol{m} \mathbf{B}(2.0 \mathrm{mg}, 0.0047 \mathrm{mmol})$, $\mathrm{Pd}_{2}(\mathrm{dba})_{3}(1.0 \mathrm{mg}, 0.0011 \mathrm{mmol}),(o-\mathrm{tol})_{3} \mathrm{P}(1.7 \mathrm{mg}, 0.0056 \mathrm{mmol})$, in toluene $/ \mathrm{Et}_{3} \mathrm{~N}$ ( $\left.3 \mathrm{~mL}, 5: 1\right)$, were reacted overnight as described in General procedure. Column chromatography (silica, 
hexanes/dichloromethane $2: 1 \rightarrow 1: 1)$ afforded a blue solid, which was washed with $\mathrm{MeOH}$ to provide a blue-green solid (2.6 mg, 68\%). ${ }^{1} \mathrm{H}$ NMR $\left(\mathrm{CDCl}_{3}, 400 \mathrm{MHz}\right) \delta-1.65$ (bs, $\left.2 \mathrm{H}\right),-1.30$ (bs, 2H), 2.02 (s, 12H), 2.12 (s, 12H), 4.41 (s, 4H), 4.52 (s, 6H), 5.07 (s, 4H), 8.69 (dd, $J=2.0$ $\mathrm{Hz}, J=4.1,2 \mathrm{H}), 8.72(\mathrm{~s}, 2 \mathrm{H}), 8.74(\mathrm{~s}, 2 \mathrm{H}), 8.90(\mathrm{dd}, J=2.0 \mathrm{~Hz}, J=4.6 \mathrm{~Hz}, 2 \mathrm{H}), 8.92(\mathrm{dd}, J=$ $2.0 \mathrm{~Hz}, J=4.4 \mathrm{~Hz}, 2 \mathrm{H}), 9.62(\mathrm{dd}, J=1.8 \mathrm{~Hz}, J=4.4 \mathrm{~Hz}, 2 \mathrm{H})$. MS $m / z[\mathrm{M}+\mathrm{H}]^{+}$Calcd for $\mathrm{C}_{52} \mathrm{H}_{54} \mathrm{~N}_{8} \mathrm{O}_{2}$ 823.4442; Found (HRMS-ESI) 823.4477.

3-Bromo-5-methoxy-8,8,18,18-tetramethyl-13-phenylethynylbacteriochlorin (5). A mixture of 3,13-dibromo-5-methoxy-8,8,18,18-tetramethyl-bacteriochlorin (4) (40 mg, 71.6 $\mu \mathrm{mol})$, phenylacetylene $(7.87 \mu \mathrm{L}, 71.6 \mu \mathrm{mol})$, potassium carbonate $(99 \mathrm{mg}, 716 \mu \mathrm{mol})$, and $\mathrm{Pd}\left(\mathrm{PPh}_{3}\right)_{4}(9.93 \mathrm{mg}, 8.59 \mu \mathrm{mol})$ in DMF $(10 \mathrm{~mL})$ was reacted at $80^{\circ} \mathrm{C}$ as described in the General Procedure. After approximately $7 \mathrm{~h}$, the mixture was diluted with ethyl acetate, washed (water and brine), and dried $\left(\mathrm{Na}_{2} \mathrm{SO}_{4}\right)$, and concentrated. The residue was purified with column chromatography (silica, hexane/ $\mathrm{CH}_{2} \mathrm{Cl}_{2}(2: 1)$ ) to afford a green solid (32 $\mathrm{mg}, 76 \%$ ). ${ }^{1} \mathrm{H}$ NMR $\left(\mathrm{CDCl}_{3}, 400 \mathrm{MHz}\right) \delta-1.89$ (s, 1H), -1.67 (s, 1H), 1.93-1.95 (two singlet, 12H), 4.34 (s, 3H), 4.42 (s, 2H), $4.43(\mathrm{~s}, 2 \mathrm{H}), 7.45-7.54(\mathrm{~m}, 3 \mathrm{H}), 7.88-7.90(\mathrm{~m}, 2 \mathrm{H}), 8.50(\mathrm{~s}, 1 \mathrm{H}), 8.58(\mathrm{~s}, 1 \mathrm{H}), 8.71\left(\mathrm{~d},{ }^{3} J\right.$ $=4.0 \mathrm{~Hz}, 1 \mathrm{H}), 8.82\left(\mathrm{~d},{ }^{3} \mathrm{~J}=4.0 \mathrm{~Hz}, 1 \mathrm{H}\right), 8.95(\mathrm{~s}, 1 \mathrm{H}) ;{ }^{13} \mathrm{C} \mathrm{NMR}\left(\mathrm{CDCl}_{3}, 100 \mathrm{MHz}\right) \delta 30.9,31.1$, 45.7, 47.6, 51.7, 64.6, 84.6, 96.4, 96.6, 97.7, 105.5, 116.8, 123.7, 124.4, 125.2, 126.7, 128.7, $131.9,134.3,135.5,135.7,138.3,154.9,161.3,170.0,170.1 ; \mathrm{MS} m / z[\mathrm{M}+\mathrm{H}]^{+}$Calcd for $\mathrm{C}_{33} \mathrm{H}_{31} \mathrm{BrN}_{4} \mathrm{O}$ 578.1676; Found (HRMS-ESI) 578.1675.

\section{5-Methoxy-8,8,18,18-tetramethyl-3-(triisopropylsilylethynyl)-13-}

phenylethynylbacteriochlorin $\quad\left(\mathbf{B C}-\boldsymbol{\beta}_{\mathbf{3}} \mathbf{B}\right)$. A mixture of $\quad \mathbf{5} \quad(32 \quad \mathrm{mg}, \quad 55 \mu \mathrm{mol})$, triisopropylsilylacetylene $(20 \mu \mathrm{L}, 110 \mu \mathrm{mol})$, and $\left(\mathrm{PPh}_{3}\right)_{2} \mathrm{PdCl}_{2}(3.87 \mathrm{mg}, 5.87 \mu \mathrm{mol})$ in DMF 6 $\mathrm{mL})$ and $\mathrm{Et}_{3} \mathrm{~N}(3 \mathrm{~mL})$ was reacted at $80^{\circ} \mathrm{C}$ as described in General Procedure. After $16 \mathrm{~h}$, the mixture was diluted with ethyl acetate, washed (water and brine), dried $\left(\mathrm{Na}_{2} \mathrm{SO}_{4}\right)$ and concentrated. The residue was purified with column chromatography [silica, hexane $/ \mathrm{CH}_{2} \mathrm{Cl}_{2}$ (2:1)] to afford a green solid (17 mg, 47\%). ${ }^{1} \mathrm{H}$ NMR $\left(\mathrm{CDCl}_{3}, 400 \mathrm{MHz}\right) \delta-1.83(\mathrm{~s}, 1 \mathrm{H}),-1.57(\mathrm{~s}$, 1H), 1.34-1.37 (m, 21H), 1.92-1.94 (two singlet, 12H), 4.41 (s, 2H), 4.41 (s, 2H), $4.43(\mathrm{~s}, 3 \mathrm{H})$, 7.44-7.54 (m, 3H), 7.88-7.90 (m, 2H), $8.50(\mathrm{~s}, 1 \mathrm{H}), 8.54(\mathrm{~s}, 1 \mathrm{H}), 8.79(\mathrm{~s}, 1 \mathrm{H}), 8.80(\mathrm{~s}, 1 \mathrm{H}), 8.91$ (s, $1 \mathrm{H}) ; 13 \mathrm{C} \mathrm{NMR}\left(\mathrm{CDCl}_{3}, 100 \mathrm{MHz}\right) \delta 11.9,19.1,31.0,31.1,45.6,45.7,47.6,51.8,64.8,84.7$, 95.5, 96.3, 96.5, 96.9, 97.5, 104.2, 113.0, 116.7, 123.7, 125.0, 126.4, 128.7, 131.4, 131.9, 134.2, 135.8, 138.2, 155.0, 161.2, 169.9, 170.3; MS $m / z[\mathrm{M}+\mathrm{H}]^{+}$Calcd for $\mathrm{C}_{44} \mathrm{H}_{52} \mathrm{~N}_{4} \mathrm{OSi} 680.3905$; Found (HRMS-ESI) 680.390512.

$\mathbf{B C}_{2}-\boldsymbol{\beta}_{3} \mathbf{B}$. A mixture of BC- $\boldsymbol{\beta}_{3} \mathbf{B}(5 \mathrm{mg}, 7.34 \mu \mathrm{mol})$, TBAF (1M in THF, $\left.15 \mu \mathrm{L}, 15 \mu \mathrm{mol}\right)$ and $\left(\mathrm{PPh}_{3}\right)_{2} \mathrm{PdCl}_{2}(2.58 \mathrm{mg}, 3.68 \mu \mathrm{mol})$ in THF $(2 \mathrm{~mL})$ and $\mathrm{Et}_{3} \mathrm{~N}(1.0 \mathrm{~mL})$ was reacted at room temperature as described in General Procedure for $18 \mathrm{~h}$. The reaction mixture was concentrated, and the residue was purified with column chromatography (silica, hexane $/ \mathrm{CH}_{2} \mathrm{Cl}_{2}(2: 1)$ ) to afford a red solid (3.4 mg, $44 \%){ }^{1} \mathrm{H}$ NMR $\left(\mathrm{CDCl}_{3}, 400 \mathrm{MHz}\right) \delta-1.45(\mathrm{~s}, 2 \mathrm{H}),-1.31(\mathrm{~s}, 2 \mathrm{H}), 1.96$. 1.98 (two singlet, 24H), 4.42, (s, 4H), 4.47 (s, 4H), 4.65 (s, 6H), $7.46-7.55$ (m, 6H), $7.89-7.91$ 
(m, 4H), $8.52(\mathrm{~s}, 2 \mathrm{H}), 8.54(\mathrm{~s}, 2 \mathrm{H}), 8.79(\mathrm{~d}, J=4.0 \mathrm{~Hz}, 2 \mathrm{H}), 8.87$ (d, $J=4.0 \mathrm{~Hz}, 2 \mathrm{H}), 8.89$ (s, $2 \mathrm{H}) ; \mathrm{MS} m / z[\mathrm{M}+\mathrm{H}]^{+}$Calcd for $\mathrm{C}_{70} \mathrm{H}_{62} \mathrm{~N}_{8} \mathrm{O}_{2}$ 1046.4990, Found HRM-ESI 1046.4987.

\section{Absorption and Fluorescence Spectra of Dyads and Benchmarks in Benzonitrile.}

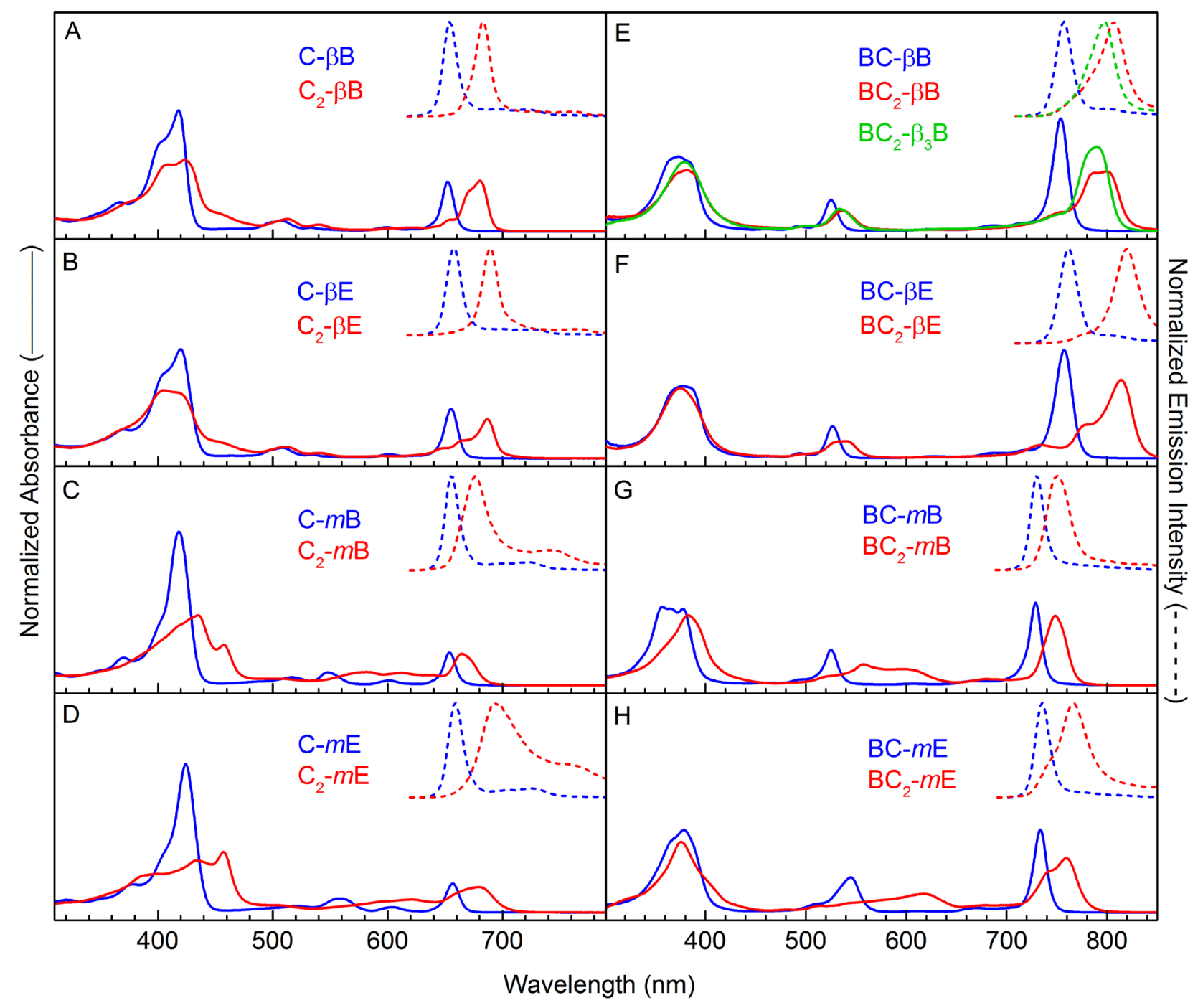

Figure S1. Absorption (solid) and fluorescence spectra (dashed) of the chlorin (left) and the bacteriochlorin (right) benchmarks (blue) and dyads (red, green) in benzonitrile. Absorption spectra were normalized to the total integrated intensity (300-1000 nm for spectra plotted vs $\mathrm{cm}^{-1}$ ) and all fluorescence spectra (500-550 excitation) to the peak intensity. 
Table S1. Spectral Characteristics of Hydroporphyrin Dyads and Benchmarks in Benzonitrile. $^{\mathrm{a}}$

\begin{tabular}{|c|c|c|c|c|c|c|}
\hline Cmpd & $\lambda_{\mathrm{B}}(\mathrm{nm})$ & $\lambda_{\mathrm{Q} 1}(\mathrm{~nm})$ & $\lambda_{\mathrm{Q} 2}(\mathrm{~nm})$ & $\lambda_{\mathrm{em}}(\mathrm{nm})$ & $\begin{array}{l}\text { fwhm em } \\
\text { (nm) }\end{array}$ & $\begin{array}{c}\text { Stokes' } \\
\text { shift }(\mathrm{nm})\end{array}$ \\
\hline \multicolumn{7}{|l|}{ Chlorins } \\
\hline C- $\beta B$ & 417 & 532 & 653 & 652 & 13 & -1 \\
\hline $\mathrm{C}_{2}-\boldsymbol{\beta} B$ & 422,452 & $538, \mathrm{n} / \mathrm{a}$ & 672,681 & 681 & 13 & 0 \\
\hline $\mathbf{C}-\boldsymbol{\beta} \mathbf{E}$ & 418 & 533 & 656 & 656 & 13 & 0 \\
\hline $\mathrm{C}_{2}-\boldsymbol{\beta E}$ & 417,452 & $538, \mathrm{n} / \mathrm{a}$ & 664,687 & 687 & 13 & 0 \\
\hline$C-m B$ & 414 & 546 & 654 & 655 & 13 & 1 \\
\hline$C_{2}-m B$ & 434,457 & 578,612 & 663,671 & 673 & 21 & 2 \\
\hline$C-m E$ & 421 & 555 & 656 & 658 & 13 & 2 \\
\hline $\mathrm{C}_{2}-\mathrm{mE}$ & 430,455 & 594,620 & 668,680 & 689 & 30 & 9 \\
\hline \multicolumn{7}{|c|}{ Bacteriochlorins } \\
\hline BC- $\beta$ B & 383 & 525 & 754 & 757 & 19 & 3 \\
\hline $\mathrm{BC}_{2}-\boldsymbol{\beta B}$ & $383, \mathrm{n} / \mathrm{a}$ & $537, \mathrm{n} / \mathrm{a}$ & 786,801 & 807 & 30 & 6 \\
\hline $\mathrm{BC}_{2}-\boldsymbol{\beta}_{3} \mathrm{~B}$ & $380, \mathrm{n} / \mathrm{a}$ & $534, \mathrm{n} / \mathrm{a}$ & 782,790 & 797 & 30 & 7 \\
\hline BC- $\beta \mathbf{E}$ & 384 & 527 & 758 & 761 & 21 & 3 \\
\hline $\mathrm{BC}_{2}-\boldsymbol{\beta E}$ & $375, \mathrm{n} / \mathrm{a}$ & 530,541 & 779,814 & 820 & 29 & 6 \\
\hline BC-mB & 378 & 525 & 729 & 730 & 16 & 1 \\
\hline $\mathrm{BC}_{2}-m \mathrm{~B}$ & $383, \mathrm{n} / \mathrm{a}$ & 557,598 & 749,753 & 749 & 27 & -4 \\
\hline BC-mE & 378 & 545 & 734 & 735 & 17 & 1 \\
\hline $\mathrm{BC}_{2}-m \mathrm{E}$ & $376, \mathrm{n} / \mathrm{a}$ & 579,617 & 742,760 & 766 & 24 & 6 \\
\hline
\end{tabular}

${ }^{\mathrm{a}}$ The bands of the benchmark monomers are listed as $\mathrm{B}, \mathrm{Q}_{1}$, and $\mathrm{Q}_{2}$ bands. For all the benchmarks but $\mathbf{C}-\boldsymbol{m} \mathbf{E}, \mathrm{Q}_{1}$ band is $\mathrm{Q}_{\mathrm{x}}$, and $\mathrm{Q}_{2}$ is $\mathrm{Q}_{\mathrm{y}}$. For $\mathbf{C}-\boldsymbol{m} \mathbf{E}, \mathrm{Q}_{1}$ is $\mathrm{Q}_{\mathrm{y}}$ and $\mathrm{Q}_{2}$ is $\mathrm{Q}_{\mathrm{x}}$. The bands of the the dyads are listed as $\mathrm{B}_{\alpha}, \mathrm{B}_{\beta}, \mathrm{Q}_{1 \alpha}, \mathrm{Q}_{1 \beta}, \mathrm{Q}_{2 \alpha}$, and $\mathrm{Q}_{2 \beta}$, where $\alpha$ is the higher energy band and $\beta$ is the lower energy one. 


\section{Resolution Enhanced Absorption Spectra of Dyads}

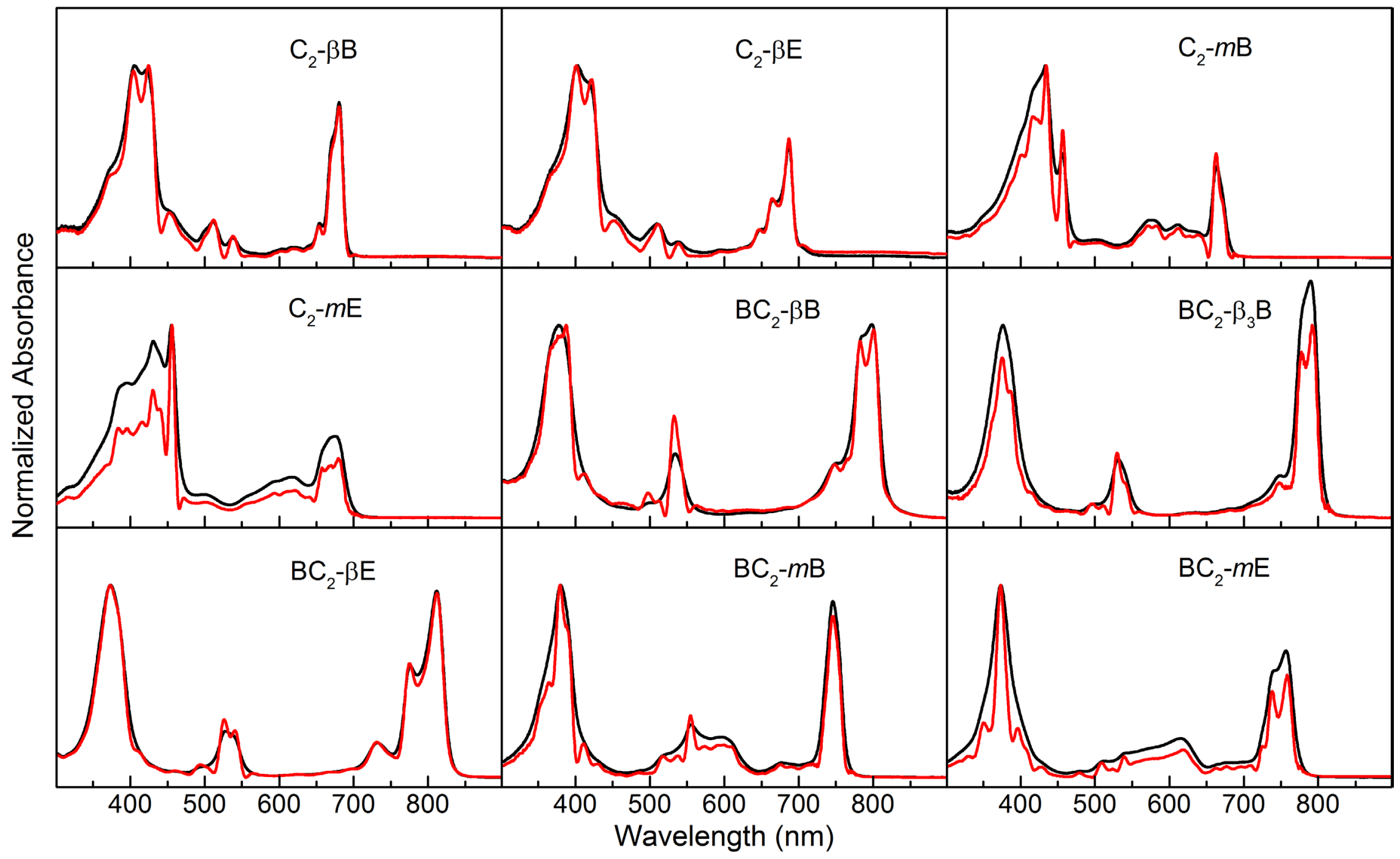

Figure S2. Original (black) and resolution-enhanced absorption spectra (red) of the hydroporphyrin dyads in toluene. All absorption spectra were normalized to at the maximum intensity in the Soret region. 
4. MO Chacteristics of $\mathrm{BC}_{2}-\boldsymbol{\beta}_{3} \mathrm{~B}$

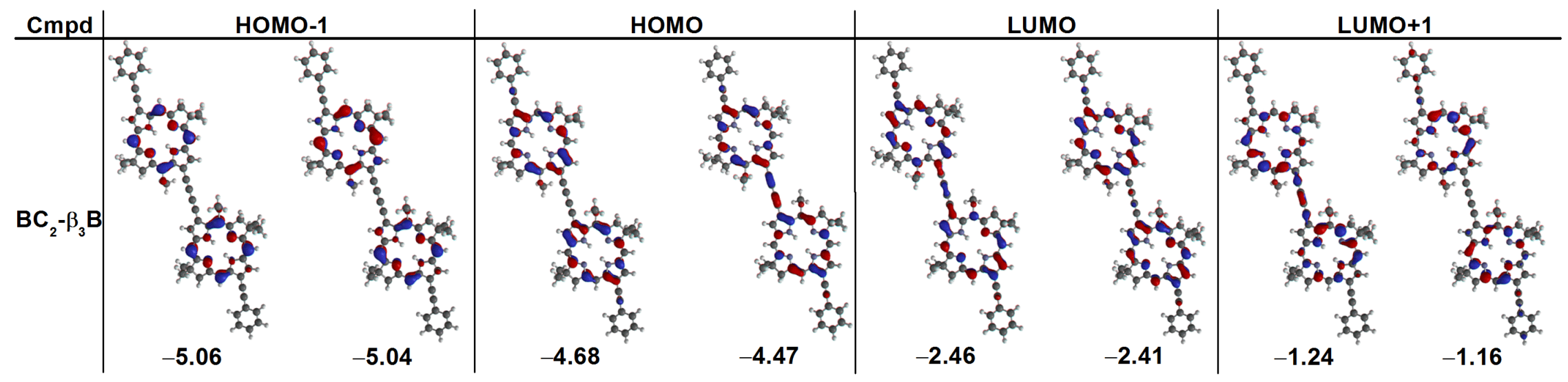

Figure S3. Frontier MOs and MO energy levels of the 3-3'-linked bacteriochlorin dyad $\mathbf{B C}_{\mathbf{2}}-\boldsymbol{\beta}_{\mathbf{3}} \mathbf{B}$. 


\section{Full Citation for References with Over Ten Authors}

8. $\quad$ Kee, H. L.; Kirmaier, C.; Tang, Q.; Diers, J. R.; Muthiah, C.; Taniguchi, M.; Laha, J. K.; Ptaszek, M.; Lindsey, J. S.; Bocian, D. F.; Holten, D. Effects of Substituents on Synthetic Analogs of Chlorophylls. Part 2: Redox Properties, Optical Spectra and Electronic Structure. Photochem. Photobiol. 2007, 83, 1125-1143.

11. Faries, K. M.; Diers, J. R.; Springer, J. W.; Yang, E.; Ptaszek, M.; Lahaye, D.; Krayer, M.; Taniguchi, M.; Kirmaier, C.; Lindsey, J. S.; Bocian, D. F.; Holten, D. Photophysical Properties and Electronic Structure of Chlorin-Imides: Bridging the Gap Between Chlorins and Bacteriochlorins. J. Phys. Chem. B 2015, 119, 7503-7515.

12. Vairaprakash, P.; Yang, E.; Sahin, T.; Taniguchi, M.; Krayer, M.; Diers, J. R.; Wang, A.; Niedzwiedzki, D. M.; Kirmaier, C.; Lindsey, J. S.; Bocian, D. F.; Holten, D. Extending the Short and Long Wavelength Limits of Bacteriochlorin Near-Infrared Absorption via Dioxo- and Bisimide-Functionalization. J. Phys. Chem. B 2015, 119, 4382-4395.

25. Kuimova, M. K.; Hoffmann, M.; Winters, M. U.; Eng, M.; Balaz, M.; Clark, I. P.; Collins, H. A.; Tavender, S. M.; Wilson, C. J.; Albinsson, B.; Anderson, H. L.; Parker, A. W.; Phillips, D. Determination of the Triplet State Energies of a Series of Conjugated Porphyrin Oligomers. Photochem. Photobiol. Sci. 2007, 6, 675-682.

40. Except for molecular mechanics and semi-empirical models, the calculation methods used in Spartan '10 have been documented in: Shao, Y.; Molnar, L. F.; Jung, Y.; Kussmann, J.; Ochsenfeld, C.; Brown, S. T.; Gilbert, A. T. B.; Slipchenko, L. V.; Levchenko, S. V.; O’Neill, D. P.; DiStasio, R. A., Jr.; Lochan, R. C.; Wang, T.; Beran, G. J. O.; Besley, N. A.; Herbert, J. M.; Lin, C. Y.; Van Voorhis, T.; Chien, S. H.; Sodt, A.; Steele, R. P.; Rassolov, V. A.; Maslen, P. E.; Korambath, P. P.; Adamson, R. D.; Austin, B.; Baker, J.; Byrd, E. F. C.; Dachsel, H.; Doerksen, R. J.; Dreuw, A.; Dunietz, B. D.; Dutoi, A. D.; Furlani, T. R.; Gwaltney, S. R.; Heyden, A.; Hirata, S.; Hsu, C.-P.; Kedziora, G.; Khalliulin, R. Z.; Klunzinger, P.; Lee, A. M.; Lee, M. S.; Liang, W.-Z.; Lotan, I.; Nair, N.; Peters, B.; Proynov, E. I.; Pieniazek, P. A.; Rhee, Y. M.; Ritchie, J.; Rosta, E.; Sherrill, C. D.; Simmonett, A. C.; Subotnik, J. E.; Woodcock, H. L., III; Zhang, W.; Bell, A. T.; Chakraborty, A. K.; Chipman, D. M.; Keil, F. J.; Warshel, A.; Hehre, W. J.; Schaefer, H. F., III; Kong, J.; Krylov, A. I.; Gill, P. M. W.; Head-Gordon, M. Phys. Chem. Chem. Phys. 2006, 8, 3172-3191. 